\title{
A FORMAÇÃo DO QUÍMICO
}

\author{
Jailson B. de Andrade* \\ Instituto de Química, Universidade Federal da Bahia, Campus Univ. Ondina, 40170-290 Salvador - BA \\ Solange Cadore \\ Instituto de Química, Universidade Estadual de Campinas, CP 6154, 13084-971 Campinas - SP \\ Paulo Cezar Vieira \\ Departamento de Química, Universidade Federal de São Carlos, CP 676, 13565-905 São Carlos - SP \\ César Zucco \\ Departamento de Química, Universidade Federal de Santa Catarina, CP 476, 88040-900 Florianópolis - SC \\ Angelo C. Pinto \\ Instituto de Química, Universidade Federal do Rio de Janeiro, CT, Bloco A, Cidade Universitária, Ilha do Fundão, \\ 21949-900 Rio de Janeiro - RJ
}

\begin{abstract}
THE CHEMIST'S EDUCATION. Training of highly qualified personnel is the most transversal axis among those identified in the document "Mobilizing Axes in Chemistry", published by SBQ, in 2002, which demands to deep the discussions, initiated in the symposium "The Chemist's Education", whose main objective was to discuss the repercussions of that training in the teaching and in the industrial section. After the publication of an initial paper, a couple of meetings and workshops held in Rio de Janeiro, in December 2003, involving the national chemist community, discussed the "The role of the graduate degrees in the Chemist's Education" and "The Chemist's Education", and confirmed the need to invest in the formation of qualified human resources in profusion and in all levels. Actions to be taken were delineated. Presently, the graduate programs in Chemistry are showing expressive results. However, although these professionals are mostly absorbed by the academy (the absorption of the industry is less significant), only a few programs give special attention to the didactical and pedagogical training. The regional decentralization is actually a tendency in most programs, but the interaction academy-economic activities is still a challenge. It is necessary to establish with the industrial section, the highly qualified professional's profile in Chemistry that the industry can absorb. The undergraduation is growing in number and quality, however still excessively concentrated in the Southeast area. The national "Curricula Guidelines" for Chemistry Courses, through its flexible approach, propitiate the integral, interdisciplinary (non compartmentalized) and critical-reflexive training of the professionals as citizens and as entrepreneurs. However, deficiencies are still recognized. The evaluation system of undergraduate courses has been able to identify the most fragile modalities of courses and other indicators. It was also verified that Brazilian chemistry industry absorbs minimally the highly qualified professionals, which is attributed to the fact that the Federal Council of Chemistry is vertically organized and does not recognize the graduate degrees as professional qualification. In the conclusion, it stands out, among other aspects, the importance of the effective implantation of the national curricula guidelines, bringing out courses whose didactical and pedagogical projects may offer a solid formation in Chemistry, but comprehensive and general enough to enable the Chemistry professionals to develop varied skills.
\end{abstract}

Keywords: the Chemist's education; curricula guidelines; chemistry courses.

\section{HISTÓRICO}

Com o objetivo de contribuir para a formulação de políticas de C\&T para a área de Química, a Diretoria e o Conselho Consultivo da Sociedade Brasileira de Química, SBQ, promoveram, em 2002, um conjunto de atividades que resultou no documento denominado "Ei-

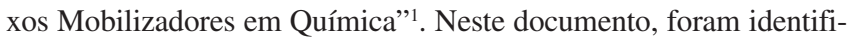
cados os seguintes eixos mobilizadores:

i) formação de recursos humanos qualificados;

ii) desconcentração regional e combate à endogenia;

iii) estímulo ao empreendedorismo e à interdisciplinaridade;

iv) aproximação pró-ativa da academia com a atividade econômica;

v) vinculação orçamentária de recursos para C\&T e

vi) combate aos gargalos institucionais.

O texto detalhado, as ações sugeridas e o valor estimado do investimento necessário estão divulgados em Química Nova ${ }^{1}$. Dentre os seis eixos identificados, o de maior transversalidade é a formação

*e-mail: jailsong@ufba.br de recursos humanos qualificados, que repercute e/ou é afetado pelos demais. Nesse sentido, para dar continuidade ao trabalho desenvolvido na formulação dos eixos, a Diretoria e o Conselho da SBQ decidiram aprofundar as discussões sobre a formação de pessoal de alto nível, iniciando pela elaboração de um documento base sobre o tema, o qual será discutido nas várias Regionais da SBQ, nas instituições de Ensino Superior e em reunião específica coordenada pela SBQ para a conclusão do documento consolidado.

\section{FORMAÇÃO DE PESSOAL DE ALTO NÍVEL}

A discussão sobre a formação de pessoal de alto nível foi iniciada no Simpósio “A FORMAÇÃO DO QUÍMICO" durante a $26^{\mathrm{a}}$ Reunião Anual da SBQ. Este Simpósio, coordenado pelo Prof. Jailson B. de Andrade (UFBA), contou com a participação dos Profs. Fernando Galembeck (UNICAMP), Roberto R. da Silva (UnB) e do Dr. Marcelo Gandur (3M do Brasil).

O objetivo principal do Simpósio foi discutir a formação dos profissionais de Química em nível de graduação e pós-graduação, 
bem como a sua repercussão no ensino (inclusive o secundário) e a inserção desses profissionais no setor industrial.

Em linhas gerais o Prof. Galembeck destacou os aspectos atuais da formação em nível superior, considerada deficiente, e a questão, de interesse muito amplo, do ensino precoce de ciências na escola primária; o Prof. Roberto abordou a formação do professor de Química do ensino médio e o Dr. Marcelo, o ambiente de trabalho do Químico na indústria. Uma sinopse do que foi discutido pelos palestrantes está publicado no Livro de Resumos da $26^{\mathrm{a}} \mathrm{RA}$ da $\mathrm{SBQ}^{2}$.

Em outubro de 2003 foi amplamente divulgado o documento preliminar "A Formação do Químico" para discussão no âmbito da Comunidade de Química. Na mensagem de encaminhamento à Diretoria e Conselho da SBQ e aos(as) Secretários(as) Regionais, o Presidente da SBQ, Prof. Paulo Cezar Vieira destacava: Estou enviando anexo o documento "A Formação do Químico" que dá continuidade às ações iniciadas com os "Eixos Mobilizadores em Química" e aprofunda a discussão sobre a formação de recursos humanos qualificados que, dentre os seis eixos identificados, é o de maior transversalidade que repercute elou é afetado pelos demais eixos. Iniciamos esta etapa com a elaboração do documento anexo, que servirá de base sobre a qual será discutido nas várias Regionais da $S B Q$, nas Instituições de Ensino Superior e em reunião específica coordenada pela $S B Q$ para a conclusão do documento consolidado. Nesse sentido, solicito ao(a) Colega que dê ampla divulgação no âmbito da Regional, bem como promova discussões e estimule sugestões e críticas.

Em 20 de novembro de 2003 foi realizada uma reunião no Centro de Gestão e Estudos Estratégicos (CGEE), em Brasília, quando foi discutido o tema A FORMAÇÃO DO QUÍMICO E O PROFISSIONAL DO FUTURO. Participaram da reunião membros da Diretoria do CGEE (Evando Mirra e Luiz Curi), o Prof. Esper Carvalheiro, representando a Academia Brasileira de Ciências e Editores e Membros da Diretoria e Conselho da SBQ.

Dois eventos ocorreram no Rio de Janeiro, em dezembro de 2003, que permitiram a consolidação do documento final: o Workshop "O PAPEL DA PÓS-GRADUAÇÃO NA FORMAÇÃO DO QUÍMICO”, coordenado pelos Profs. Vitor F. Ferreira (UFF) e Solange Cadore (UNICAMP), ocorreu em 01 e 02 de dezembro de 2003 na Universidade Federal Fluminense em Niterói e o Workshop "A FORMAÇÃO DO QUÍMICO”, coordenado pela Profa. Claudia M. Rezende (UFRJ), ocorreu em 03 de dezembro de 2003 na Universidade Federal do Rio de Janeiro. Os dois eventos tiveram ampla participação da comunidade de química, em especial coordenadores de cursos de Pós-graduação, membros dos Comitês Assessores da CAPES e do CNPq e Diretores e Conselheiros da SBQ.

Ficou patente em todas as discussões promovidas pela SBQ que é necessário investir na formação de recursos humanos qualificados em profusão e em todos os níveis. A este eixo foram associadas algumas ações como o acesso direto ao doutorado para os alunos que participaram de programas de Iniciação Científica; incentivo às atividades conjuntas de graduação e de pós-graduação; estímulo ao intercâmbio entre as IES e entre estas e as instituições de nível médio; o pós-doutorado como um processo de formação continuada; a constituição de grupo de trabalho da SBQ para acompanhar a implantação das diretrizes curriculares nas IES; o incentivo a programas para formação de docentes de 3 o grau e a realização de Semanas Nacionais de Química com a participação de estudantes do nível médio, de graduação, pós-graduação e professores, como forma de mobilização e integração entre os vários níveis de ensino.

\section{A PÓS-GRADUAÇÃO}

A Pós-graduação em Química no Brasil vai bem³ ${ }^{3}$ a formação de mestres e doutores aumenta a cada ano, o que reflete positivamente nos cursos de Graduação. Em 2001, foram titulados 418 mestres e 292 doutores.

Apesar da maior parte dos egressos da Pós-graduação possuir uma sólida formação em Química e serem bons pesquisadores, somente uma pequena parcela desses doutores é absorvida pela indústria. $\mathrm{O}$ que se observa, cada vez mais, é que os cursos de Graduação em Química do país, sejam eles da rede pública ou privada, têm nesses jovens doutores uma fonte de revitalização de seus quadros. Paradoxalmente, porém, são poucos os Programas que possuem um elenco de disciplinas voltadas à formação didático-pedagógica dos mestrandos/doutorandos. Mesmo não atendendo sempre às necessidades e expectativas, os programas voltados à atuação dos alunos em atividades de capacitação docente têm estimulado esta prática, oferecendo uma oportunidade para os pós-graduandos exercerem uma atividade complementar à pesquisa. Aos poucos se observa, em diversas Instituições, o desenvolvimento de dissertações e teses tendo o Ensino de Química como linha de pesquisa, o que contribuirá para melhorar ainda mais a atuação dos jovens doutores no ensino de Graduação.

O surgimento de novos programas de pós-graduação (recentemente a CAPES aprovou 5 novos cursos de Mestrado) leva em um primeiro momento, à qualificação de recursos humanos. Paralelamente, a criação de novos cursos leva à desconcentração regional e, através da absorção dos novos doutores, combate-se a endogenia. Programas envolvendo diferentes IES do país, como o PROCAD, são importantes porque estimulam a formação e a qualificação de recursos humanos. Esses jovens pesquisadores sentem que, hoje, a interdisciplinaridade e a interação com o setor industrial são essenciais. Não é suficiente a transmissão do conhecimento compartimentalizado nem dissociado da realidade do mercado de trabalho. Mas, a interação academia-empresa ainda é um desafio que necessita ser enfrentado, apesar do número crescente de mestres que atuam nesse mercado. Nesse sentido, é preciso divulgar os trabalhos desenvolvidos na Academia junto ao setor industrial, destacar as potencialidades da interação em pesquisa, bem como a participação daquele setor no estabelecimento do perfil do profissional em Química, altamente qualificado, que este segmento da sociedade pode absorver.

\section{GRADUAÇÃO}

Uma visão geral da situação dos cursos de graduação em química revela que a região sudeste concentra $41 \%$ dos cursos, ao passo que as regiões norte e centro-oeste representam, em conjunto, $10 \%$ do sistema (Figura 1). Em 2001, estes cursos ofereceram cerca de 6.400 vagas, matricularam em torno de 18.000 alunos e titularam aproximadamente 2.000 profissionais. Como mostra a Tabela 1, o número de cursos de Química nos últimos 6 anos cresceu significativamente, embora não no ritmo quase desenfreado de várias outras áreas (ver expansão do ensino superior, Jornal a "Folha de S. Paulo", 03/08/2003). As IES Federais (44\%) e as particulares (36\%) lideram as matrículas (Figura 2).

\section{DIRETRIZES CURRICULARES PARA OS CURSOS DE QUÍMICA}

As Diretrizes Curriculares para os Cursos de Química, elaboradas em atendimento à nova Lei de Diretrizes e Bases da Educação Nacional promulgada em 1996 (Lei 9.394/96) e ao Edital no 04/97 da Secretaria de Educação Superior do $\mathrm{MEC}^{4}$, estabelecem, como princípio, a flexibilização curricular que, sem prejuízo de uma formação didática, científica e tecnológica sólida, avance também na direção de uma formação humanística que dê condições ao egresso de exercer a profissão em defesa da vida, do ambiente e do bem-estar dos cidadãos. Espera-se que os novos currículos ofereçam mais do 


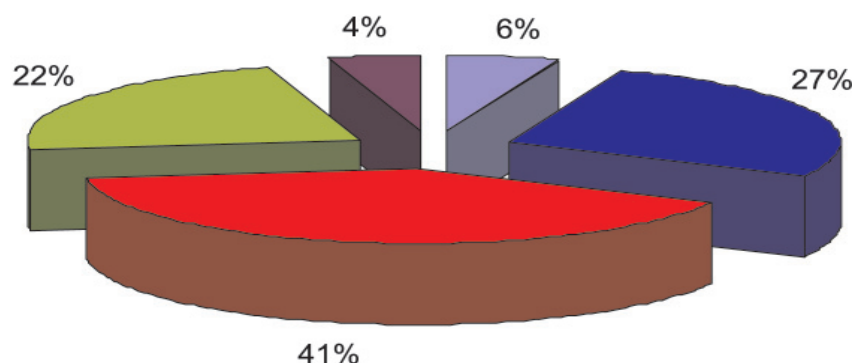

$\square$ NORTE $\square$ NE $\square$ SUDESTE $\square$ SUL $\square$ CO

Figura 1. Distribuição dos cursos de Graduação em Química por Região do país

Tabela I. Evolucão dos Cursos de Química

\begin{tabular}{lrrr}
\hline & 1966 & 1998 & 2001 \\
\hline $\mathrm{N}^{\circ}$ Cursos & 83 & 89 & 99 \\
Vagas oferecidas & 5.395 & 5.737 & 6.371 \\
Candidatos & 12.428 & 15.944 & 21.645 \\
Ingressos & 4.055 & 4.607 & 5.424 \\
Matrículas & 14.742 & 15.004 & 18.224 \\
Concluintes & 1.437 & 1.445 & 2.037 \\
\hline
\end{tabular}
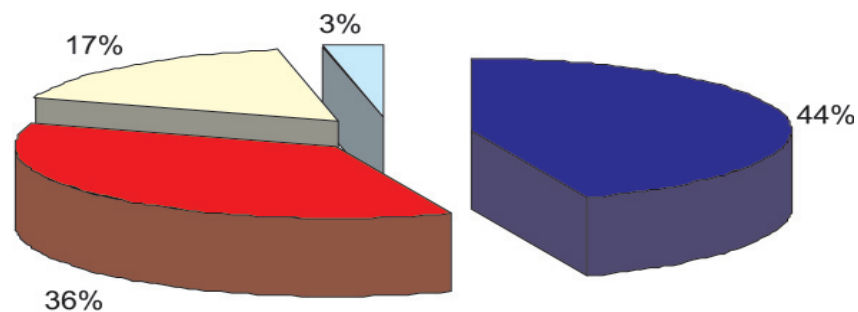

Federais $\square$ Particulares $\square$ Estaduais $\square$ Municipais

Figura 2. Distribuição dos cursos de Graduação em Química nas IES do país

que o domínio cognitivo dos conteúdos, contemplando atividades que visem estabelecer correlações entre áreas, ampliando o caráter interdisciplinar. Além disso, espera-se que o professor, mais que a fonte principal de informações para os estudantes, seja um sistematizador e facilitador de idéias.

De acordo com as Diretrizes, o mais importante em um currículo não é a quantidade de disciplinas, mas sim a sua articulação em torno de uma proposta de ensino na qual estejam definidos claramente os objetivos do curso e a sua abrangência. Dessa forma, estimula-se o caráter crítico-reflexivo dos estudantes buscando a formação de profissionais que venham a gerar empregos e não que visem apenas a ser empregados.

Na elaboração de seus currículos, é recomendável que os cursos evitem pulverizá-los com exagerado número de disciplinas que segmentam o conhecimento da área e deixam de ressaltar o essencial do campo de conhecimento. Além disso, a compartimentalização leva à repetição de conteúdos de maneira desnecessária. Mais do que o domínio cognitivo do conteúdo de Química, espera-se que os novos currículos contemplem atividades que visem estabelecer correlações entre a Química e áreas conexas, ampliando o caráter interdisciplinar. Assim, os currículos buscarão integração entre os conteúdos básicos e os conteúdos profissionais essenciais e promoverão, também, através de seus planos de ensino, condições reais e quantitativamente significativas de integração de atividades e experiências práticas em laboratórios e estágios. Nesse sentido foi proposta a seguinte composição para o quadro curricular:

i) conteúdos básicos essenciais, envolvendo teoria e laboratório e dos quais deverão fazer parte: Matemática, Física e Química;

ii) conteúdos profissionais essenciais para o desenvolvimento de competências e habilidades;

iii) conteúdos complementares essenciais para a formação humanística, interdisciplinar, gerencial e

iv) atividades extra-classe.

Uma das expectativas com os novos currículos é a ampliação dos conteúdos em áreas correlatas (como, por exemplo, a Biologia) além de outras áreas de importância na formação profissional, tais como Eletrônica, Legislação, História, etc., que possam estimular o empreendedorismo (Figura 3). Essas expectativas foram bem registradas nos dez mandamentos do universitário do século XXI, resumo extraído da Conferência Mundial de Ensino Superior da UNESCO, que ocorreu em Paris, em 1998 (Quadro 1).

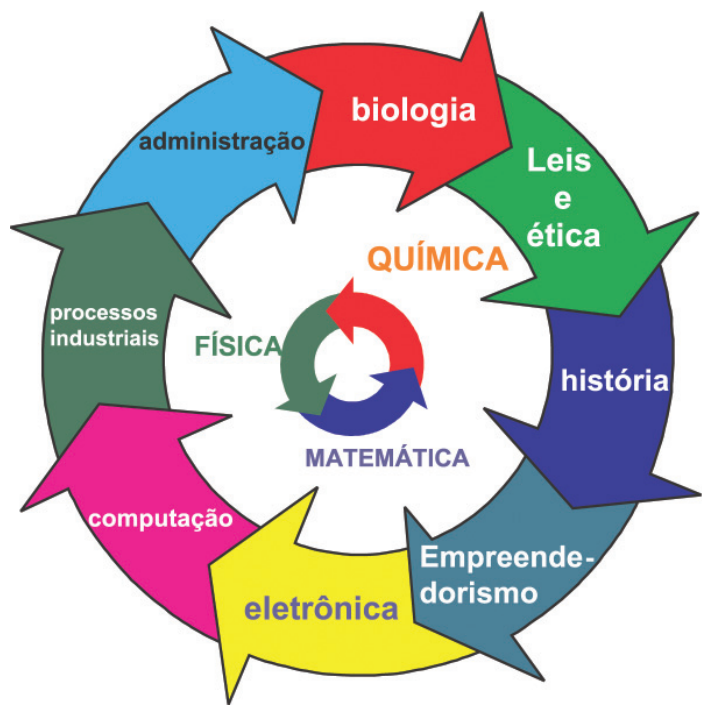

Figura 3. Conteúdos sugeridos na formação dos Químicos

Quadro 1. Dez mandamentos do Universitário do século XXI (Conferência Mundial sobre o Ensino Superior - UNESCO, 1998)

1. Seja flexível, não se especialize demais;

2. Invista na criatividade, não só no conhecimento;

3. Aprenda a lidar com incertezas (o mundo é assim);

4. Prepare-se para estudar durante toda a vida;

5. Tenha habilidades sociais e capacidade de expressão;

6. Saiba trabalhar em grupo, bons empregos exigem isso;

7. Esteja pronto para assumir responsabilidades;

8. Busque ser empreendedor, crie o seu emprego;

9. Entenda as diferenças culturais (o trabalho globalizou);

10. Adquira intimidade com novas tecnologias.

Em trabalho recente ${ }^{5}$, sob os auspícios da UNESCO, foi realizada uma revisão dos currículos dos cursos de Química de várias universidades latino americanas. Nesta está registrado um dos exemplos do reconhecimento da importância das Diretrizes Curriculares para os Cursos de Química, elaboradas no Brasil: 


\begin{abstract}
" Los cambios señalados en el mercado del trabajo plantean una modificación central tanto en los aspectos relacionados con la equidad, como en aquellos referentes a la competitividad. Se trata del paso de las calificaciones a las competencias. Lo anterior para el caso de la enseñanza universitaria de la química está recogido en el documento brasileño Diretrizes curriculares Nacionais para os cursos de Química (Apéndice 2) único en la región que plantea a nivel de ley lo que debe buscar la enseñanza en el siglo XXI. Será necesario estar al tanto de su concreción, de manera que no quede como otros documentos, sólo en eso. Con todos sus problemas Brasil ejemplifica la ambición de superar su pasado. En sus muchas y nuevas universidades ( comparadas con otras tantas del resto del continente ) se genera en términos prácticos la mitad del conocimiento químico que aporta la región, además de que es el país donde más se aplica. Hay que enseñar también, para la innovación y el cambio"s.
\end{abstract}

\section{A FORMAÇ̃̃O DO QUÍMICO HOJE}

É voz corrente que os químicos formados em várias IES públicas e privadas do país têm nível de conhecimento compatível com o de seus colegas de países desenvolvidos. Isso significa, simplesmente, que há um reconhecimento internacional e, especialmente, latino-americano, de que várias instituições no Brasil estão formando bons químicos. Todavia, não se pode ignorar que mesmo esses centros de excelência formam, sob alguns aspectos, químicos despreparados para um mundo em constante mudança.

As principais deficiências na formação dos químicos brasileiros podem ser enquadradas como: a) fraca formação em áreas afins à Química, por exemplo, Biotecnologia, Biologia e outras modalidades de conhecimento atuais e novas para os químicos; b) a maioria dos químicos é formada com orientação tipicamente voltada para uma pós-graduação como um fim em si mesma, quando esta deveria ser encarada como um meio de formação continuada, conseqüentemente para aumentar a capacidade criativa e empreendedora do candidato (70\% dos graduandos em Química querem fazer cursos avançados); c) desconhecimento da importância e da participação estratégica da Química na vida contemporânea; d) os estudantes gastam muito tempo com conteúdos de interesse restrito, ao invés de se ocuparem/aprenderem sobre as substâncias que são insumos do dia-adia das indústrias e estão presentes no cotidiano das pessoas (corantes, tensoativos, polímeros, compostos da indústria de base, especialidades da química fina); e) há desconhecimento do sistema econômico em que a Química é desenvolvida: empresas, produtos, processos e setores de aplicação e, pior, pouco se aprende, na graduação, sobre o exercício e a regulamentação da profissão do Químico f) na maioria dos projetos didático-pedagógicos dos cursos de Química, a carga horária experimental é de apenas $30 \%$.

A avaliação das condições de ensino - ACE - em 2000 (então chamada de avaliação das condições de oferta), mostrou que apenas $33 \%$ dos cursos de Química eram de Bacharelado. Àquela época, $42 \%$ dos cursos eram de Licenciatura plena e $25 \%$ de Ciências com habilitação em Química, também uma modalidade, embora em extinção, de formação de professores. Aproximadamente dois terços dos cursos em Ciências, que têm quase um quarto de todos os alunos matriculados em Cursos de formação de professores de química, estavam localizados em IES particulares geralmente no interior do País. A avaliação das Condições de Ensino considerou 3 indicadores, os quais foram divididos em vários itens: 1 . Corpo docente: titulação, dedicação ao curso, adequação do perfil, valorização docente, experiência no magistério superior e administração acadêmica do curso, titulação, dedicação ao curso, adequação do perfil docente, produção científica, plano de capacitação, plano de carreira e nível salarial (cada um desses itens se desdobra em vários indicadores). 2. Organização didáticopedagógica: estrutura curricular, atividades para o corpo discente e informações complementares sobre o corpo discente (cada um desses itens se desdobra em vários indicadores). 3. Instalações: instalações gerais, instalações especiais, biblioteca e laboratórios (cada um destes itens se desdobra em vários indicadores).

No indicador corpo docente, $11 \%$ dos cursos receberam conceito insuficiente $(\mathrm{CI}), 47 \%$ conceito regular (CR), $22 \%$ conceito bom (CB) e $20 \%$ conceito muito bom (CMB). O alto percentual de conceito regular é devido aos Cursos de Ciências que formam professores de química, porque $63 \%$ destes cursos receberam CR.

Em organização didático-pedagógica, $38 \%$ dos cursos tiveram CB e $31 \%$ CMB. No indicador instalações, $41 \%$ dos cursos receberam $\mathrm{CB}$ e $30 \% \mathrm{CMB}$. Apesar da média geral deste indicador ser satisfatória, o item biblioteca foi considerado insuficiente em $40 \%$ dos cursos.

Essa avaliação, com as devidas cautelas, revelou que os Cursos de Bacharelado tiveram o melhor desempenho dentre todas as modalidades e que os cursos de ciências dirigidos para a formação de professores de química mostraram deficiências em todos os indicadores. Outra conclusão é que os cursos de responsabilidade das IES federais e de algumas universidades estaduais são os melhores. Isso se deve certamente à qualificação do corpo docente desses cursos e do envolvimento dos seus professores com atividades científicas.

\section{A INDÚSTRIA QUÍMICA}

O Brasil lidera a indústria química na América Latina ${ }^{6}$, onde é o maior produtor de amônia, benzeno, cloro, etileno, formaldeído, polietileno, polipropileno, propileno, cloreto de polivinila, hidróxido de sódio, estireno, tolueno e uréia ${ }^{6}$. Espera-se que já em 2006 o País seja auto-suficiente em petróleo. A alta do preço do petróleo em 1973, agravada em 1979, levou a uma grande onda depressiva na economia brasileira. O setor industrial Químico no Brasil faturou de 42,2 bilhões de dólares americanos em 2003 (Figura 4) ${ }^{7}$, sendo aproximadamente metade do faturamento resultante de insumos de uso industrial e química de base (22,2 bilhões de dólares americanos). Apesar da posição de destaque, a indústria química brasileira apresenta um déficit sistêmico de cerca de 6 bilhões de dólares americanos anuais ${ }^{7}$

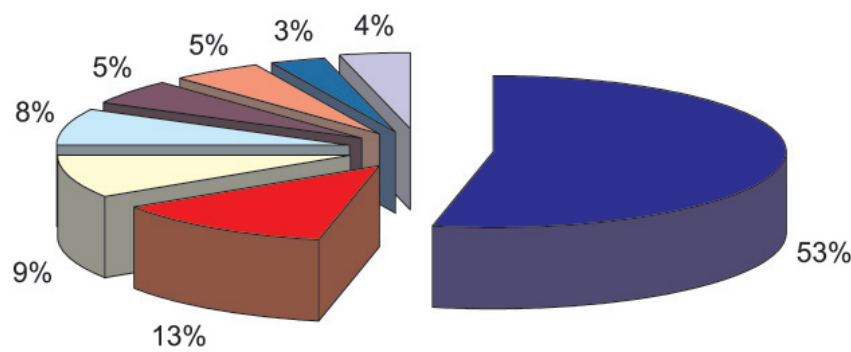

Produtos químicos de uso industrial US $\$ 22,5$

Produtos farmacêuticos US $\$ 5,3$

Adubos e fertilizantes US $\$ 3,9$

$\square$ Higiene pessoal, perfumaria e cosméticos US $\$ 3,2$

Defensivos agrícolas US\$2,3

Sabões e detergentes US\$2,1

Tintas, esmaltes e vernizes US\$1,3

$\square$ Outros US\$1,6

Figura 4. Perfil da indústria Química no Brasil 
O levantamento feito com egressos da pós-graduação constatou que cerca de $25 \%$ dos mestres e somente $5 \%$ dos doutores em Química são absorvidos pelas empresas ${ }^{8}$. Um dos gargalos para a absorção de pós-graduados em Química pelo setor industrial está relacionado ao não reconhecimento da pós-graduação como qualificação profissional pelo sistema Conselho Federal de Química (CFQ)/Conselhos Regionais de Química (CRQ).

A profissão de Químico é regulamentada pela Lei № 2.800 de 18 de junho de $1956^{\circ}$. Através da RESOLUÇÃO NORMATIVA № $36^{10}$ foi estabelecido um elenco de 16 atividades para os Profissionais da Química:

Profissional de Química - Atividades 1 a 7

Profissional de Química Tecnológica - Atividades 1 a 13

Profissional de Engenharia Química - Atividades 1 a 16

O CFQ criou um sistema de atribuições profissionais verticalizado, no qual o profissional de Química, independentemente de ser portador de diploma de pós-graduação, ocupa a base da pirâmide, sendo-lhe atribuído um menor número de atividades profissionais.

\section{CONCLUSÕES E DESAFIOS}

Nos últimos anos, por força dos processos de avaliação, o ensino superior brasileiro tem mostrado melhora. $\mathrm{O}$ número de docentes doutores nas IES tem crescido com rapidez. As condições físicas, os laboratórios, as bibliotecas, as facilidades computacionais em quase todas as instituições estão se transformando para melhor. Porém, conforme já mencionado, no que concerne à formação do Químico, várias questões permanecem inalteradas, comprometendo, definitivamente, a formação do graduado.

Com acertos e erros conceituais e metodológicos amplamente conhecidos e discutidos, os resultados das provas dos estudantes no Exame Nacional de Curso ainda não permitem um mapeamento seguro da qualidade curricular e didático-pedagógica dos cursos de Graduação em Química no Brasil. Não se pode, entretanto, deixar de perceber a importância e a gravidade de algumas informações paralelas obtidas através do questionário que os alunos respondem como parte desse Exame. A maioria dos discentes de Química que se submeteu aos ENC de 2000 a 2002 disse que: i) dedicava apenas $5 \mathrm{~h}$ semanais para estudo extra-classe; ii) sabia muito pouco de inglês e de espanhol; iii) lia, em média, um livro por ano e iv) quase não lia jornais. Todavia, $40 \%$ desses discentes disseram que pretendiam trabalhar no ensino; $70 \%$ pretendiam fazer cursos de pós-graduação e mais de $80 \%$ queriam trabalhar na área de Química.

Mudanças devem ser introduzidas nos projetos didático-pedagógicos dos cursos para que estes ofereçam uma formação sólida em Química, mas abrangente e generalista o suficiente para que o Químico possa se desenvolver em mais de uma direção. Muitos são os desafios que se nos impõem nessa tarefa de formação do Químico:

i) implantar as Diretrizes;

ii) melhorar a qualificação para a docência dos professores universitários e do ensino médio;

iii) redefinir a formação profissional buscando a formação de um graduado com intimidade com novas tecnologias e com espírito empreendedor;

iv) formar pós-graduados com possibilidade de inserção no setor industrial;

v) buscar a transformação da indústria química brasileira de indústria de base para indústria de especialidades; vi) buscar a alteração das atribuições profissionais, de modo a eliminar a verticalização de atribuições, e o reconhecimento da pósgraduação como qualificação profissional e

vii) responder à questão qual o perfil do profissional de Química que estaremos formando em 5, 10 e 20 anos? Esse é o perfil que a sociedade necessita?

\section{AGRADECIMENTOS}

À Universidade Federal Fluminense (UFF), Universidade Federal do Rio de Janeiro (UFRJ) e Centro de Gestão e Estudos Estratégicos (CGEE) pelo apoio logístico e financeiro para a realização dos workshop. Aos(Às) colegas que participaram das várias discussões sobre o tema: Adriano L. Monteiro, Aida Espínola, Alcino P. de Aguiar, A. Arnóbio de S. da Gama, André Gustavo H. Barbosa, Anna Claudia Cunha, Antonio Luiz Braga, Antonio S. Mangrich, Benedito dos S. Lima Neto, Bianca Machado, Carlos Alberto L. Filgueiras, Carmem Lúcia de P. e S. Zonta, Claudia M. Rezende, Claudia R. Martins, Cláudio Costa Neto, Dorila Piló-Velloso, Eduardo Hollauer, Eliezer J. de L. Barreiro, Evando Mirra, Esper Carvalheiro, Faruk J. Nome Aguilera, Fernando Galembeck, Frederico G. Cruz, Germano Tremiliosi Filho, Gilberto A. Romeiro, Hans Viertler, Isabel Maria N. da S. Moreira, Jari N. Cardoso, João Batista N. da Costa, José Daniel F. Villar, José Israel Vargas, José Walkimar de M. Carneiro, Júlio Carlos Afonso, Jussara Miranda, Keiko Takashima, Ledjane S. Barreto, Luciana A. Silva, Lucidéa G. R. Coutinho, Luiz Carlos Dias, Luiz Curi, Luiz Eduardo P. Borges, Luiz Gonzaga de F. Lopes, Luiz Trugo, Mara Elisa F. Baribante, Manfredo Hörner, Marcelo Gandur, Marcelo M. Pereira, Marcos C. de Souza, Maria D. Vargas, Maria Fátima das G. F. da Silva, Mônica Regina M. P. de Aguiar, Munir S. Skaf, Oswaldo Luiz Alves, Paulo Cícero do Nascimento, Paulo Rogério P. Rodrigues, Pedro Afonso de P. Pereira, Ridvan N. Fernandes, Roberto R. da Silva, Rosane N. Castro, Rosângela de A. Epifânio, Sérgio de Paula Machado, Sergio Pinheiro, Silvia Maria Sella, Sonia S. Costa, Susana I. Córdoba de Torresi, Valfredo A. Lemos, Vanderlan da S. Bolzani, Vera Lúcia P. Pereira, Vera Lucia Pardini, Vitor Francisco Ferreira, Werner Bruce Kover, Wilson A. Lopes.

\section{REFERÊNCIAS}

1. de Andrade, J. B.; Cadore, S.; Vieira, P. C.; Zucco, C.; Pinto, A. C.; Quim. Nova, 2003, 26, 445.

2. de Andrade, J. B.; Galembeck, F.; Gandur, M. C.; Livro de Resumos da 26 Reunião Anual da SBQ, Poços de Caldas, Brasil, 2003.

3. da Gama, A. A. S.; Cadore, S.; Ferreira, V. F.; Quim. Nova 2003, 26, 618.

4. Zucco, C; Pessine, F. B. T.; de Andrade, J. B.; Quim. Nova 1999, $22,454$.

5. Chamizo, J. A.; "Situación actual y desafíos de la enseñanza universitaria de la química en America Latina" Red de Facultades de Ciencias de América Latina y el Caribe, UNESCO, 2003.

6. Tullo, A.H.; C\&EN, 2003, 81, 15.

7. www.abiquim.org.br, acessada em Janeiro 2004

8. Braga, M. M.; Azevedo, S.; Quim. Nova 2002, 25, 696

9. Lei N ${ }^{\circ} .2800$ de 18 de junho de 1956 - Cria os Conselhos Federal e Regionais de Química, dispões sobre a profissão de Química e dá outras providências.

10. Lei $\mathrm{N}^{\circ} .2800$ de 18 de junho de 1956, RESOLUÇÃO NORMATIVA Nº. 36 de 25.04.1974 - Dá atribuições aos profissionais da Química e estabelece critérios para a concessão das mesmas, em substituição à Resolução Normativa No. 26, Artigo 1o. - Estabelece um elenco de 16 atividades para os Profissionais da Química. 